\title{
Sintomas frecuentes del cáncer de ovario en la atención primaria
}

\section{Objetivo}

Identificar la frecuencia, la severidad y la duración de los síntomas asociados al cáncer de ovario en la atención primaria.

\section{Diseño}

Estudio de casos y controles.

\section{Lugar}

Dos clínicas de atención primaria (Medicina Familiar y Clínica de la Mujer) y Servicios de Ginecología de dos centros médicos universitarios de Seattle, EE.UU.

\section{Participantes}

1709 pacientes que consultaron en las clínicas de atención primaria completaron en forma anónima una encuesta sobre los síntomas experimentados durante el último año. Una encuesta idéntica fue realizada en 128 pacientes que ingresaron al Servicio de Ginecología para cirugía diagnóstica de masas ováricas o pelvianas.

\section{Evaluación de factores de riesgo}

Las participantes respondieron un cuestionario sobre 20 síntomas típicamente asociados al cáncer de ovario categorizados en 8 grupos (dolor, dificultad para comer, síntomas abdominales, urinarios, intestinales, trastornos de la menstruación, dispareunia o sangrado y síntomas constitucionales) indicando la frecuencia, la severidad y la duración. Además en el cuestionario se incluían datos como la edad, raza, paridad, nivel de educación, historia médica previa y motivo de la consulta.

\section{Medición de resultados principales}

Compararon la frecuencia, la severidad y la duración de los síntomas reportados en la encuesta entre las pacientes con cáncer de ovario y las que consultaron en las clínicas de atención primaria.

\section{Resultados principales}

En las pacientes de los centros de atención primaria, el $72 \%$ tuvieron síntomas recurrentes con un promedio de 2 síntomas. Los más comunes fueron el dolor lumbar (45\%), fatiga (34\%), flatulencia $(28 \%)$, constipación $(24 \%)$, dolor abdominal (22\%) y síntomas urinarios (16\%) con una severidad promedio $2 / 3$ en una escala 1 5 . En las pacientes con cáncer de ovario, el $94 \%$ respondieron haber tenido algún síntoma en el último año y el $67 \%$ tuvo síntomas recurrentes con un promedio de 4 años. Los síntomas más frecuentes fueron el incremento de diámetro abdominal [OR 7,4 IC95\% 3,8-14,2], flatulencia [OR 3,6 IC95\% 1,8-7,0], urgencia miccional [OR 2,5 IC95\% 1,3-4,8] y dolor pelviano [OR 2,2 IC95\% 1,2$3,9]$.

En promedio las pacientes con cáncer de ovario tuvieron 8 síntomas en comparación con las de atención primaria que tuvieron 4 , la duración promedio de los síntomas fue de 3-6 meses vs. 12-24 meses.

La coexistencia de los síntomas de flatulencia, el incremento del diámetro abdominal y los síntomas urinarios se asoció significativamente al cáncer de ovario en comparación con los tumores benignos y el grupo control [OR 5,3 IC95\% 2,2-12,6 y OR 9,4 IC $95 \%$ 5,017,7 respectivamente].

\section{Conclusión}

Los síntomas de mayor severidad y frecuencia que lo habitualmente esperado y aquellos de reciente comienzo necesitarían mayor evaluación diagnóstica debido a su asociación con las masas ováricas benignas y malignas.

Fuente de financiamiento:Ovarian Cancer Research Fund Inc, New York, NY

\section{Comentario}

El cáncer de ovario es la quinta causa de muerte por cáncer en las mujeres y se estima que su prevalencia es de 30 a 50 por cada 100.000 mujeres, su incidencia es de 14 por cada 100.000 mujeres por año. ${ }^{1}$

En la mayoría de los casos la sobrevida a los 5 años es inferior al $30 \%$ en el momento del diagnóstico. Pero esta tasa puede alcanzar $80-90 \%$ para el estadio I, lo cual indica que la detección temprana es un factor fundamental para reducir la mortalidad por esta enfermedad.

Sin embargo la evidencia actual indica que si bien los métodos de screening (el dosaje de CA-125 y la ecografía transvaginal) tienen una alta sensibilidad y especificidad para detectar al cáncer de ovario, los potenciales daños secundarios (necesidad de procedimientos invasivos para la confirmación diagnóstica y el impacto psicológico de los resultados falsos positivos) sobrepasan a los beneficios potenciales. Por lo tanto, muchas organizaciones, incluyendo American Cancer Society, U.S. Preventive Services Task Force y Canadian Task Force on Preventive Health Care recomiendan no realizar rastreo de rutina en población general (Recomendación D)..$^{2 \cdot 3}$

Clásicamente, incluyendo la mayoría de los textos de medicina, el cáncer de ovario es conocido como "enfermedad silente (silent killer)" debido a su característica de no presentar síntomas típicos hasta que progresa a estadios mas avanzados, por lo tanto, entraña cierta dificultad su diagnóstico en estadios tempranos. Sin embargo algunos pequeños estudios retrospectivos han señalado que muchas pacientes presentan síntomas previos al diagnóstico aunque estos no son necesariamente ginecológicos. En estos estudios se observó que el $95 \%$ de pacientes con cáncer de ovario tenía síntomas previos a su diagnóstico y los más frecuentes fueron dolor, síntomas relacionados al tracto gastrointestinal, síntomas urinarios y constitucionales. ${ }^{4 \cdot 5}$

\section{Conclusiones del comentador}

Es importante enfatizar que la mayoría de las mujeres que consultan por estos síntomas no presentarán cáncer de ovario. Pero si tenemos en cuenta que por el momento el rastreo de cáncer de ovario en la población general no está recomendado, este estudio tiene una implicancia clínica importante aportando una mejor definición de los síntomas típicamente asociados, que ayudarán a identificar síntomas y signos tempranos para reducir la mortalidad por esta enfermedad.

\section{Dra. Chu Yoon [ UMF y P. Hospital Italiano de Buenos Aires. ]}

Yoon, C. Síntomas frecuentes del cáncer de ovario en la atención primaria. Evid. actual. práct. ambul.2004;7:142. Comentado de: Frequency of symptoms of ovarian cancer in women presenting to primary care clinics. Goff B, Mandel L, Melancon C, Muntz H. JAMA. June 9, 2004;291:2705-2712

Referencias

1.Epithelial Ovarian Cancer : Prevention, Diagnosis, and Treatment. CA Cancer J Clin 1999;49:297-320.

2.Cancer Screening Guidelines. Am Fam Physician 2001;63:1101-12.

3.American College of Preventive Medicine Practice Policy Statement. Screening Asymptomatic Women for Ovarian Cancer.

4.Goff BA, Mandel L, Muntz HG, Melancon CH. Ovarian cancer diagnosis: results of a national ovarian cancer survey. Cancer. 2000;89:2068-2075.

5. Olson SH, Mignone L, Nakraselve C, Caputo TA, Barakat RR, Harlap S. Symptoms of ovarian cancer. Obstet Gynecol. 2001;98:212-7. 